\title{
Effect of Reinnervation on the Degradation Rate of Junctional Acetylcholine Receptors Synthesized in Denervated Skeletal Muscles
}

\author{
S.-L. Shyng and M. M. Salpeter \\ Section of Neurobiology and Behavior, Cornell University, Ithaca, New York 14853
}

Two populations of ACh receptors (AChRs) with different degradation rates have been shown to coexist in the postsynaptic membrane after denervation of the neuromuscular junction (NMJ). One population, consisting of the slowly degrading original AChRs inserted into the plasma membrane prior to denervation, has a degradation half-life $\left(t_{1 / 2}\right)$ of $\sim 8$ d. This degradation rate accelerates after denervation (to a $t_{1 / 2} \sim 3 \mathrm{~d}$ ), but can be decelerated back to the predenervation rate by reinnervation. The second population, the rapidly degrading new AChRs, which replace the degrading original AChRs at the NMJ after denervation, resembles embryonic AChRs, with a $t_{1 / 2}$ of $\sim 1 \mathrm{~d}$. In the present study, we report that the degradation rate of these new junctional AChRs is unaltered for 3-6 half-lives after reinnervation. We further report that a small amount $(<10 \%)$ of slowly degrading AChRs ( $t_{1 / 2} \sim 3$ d) may also be synthesized in denervated muscle. We suggest that, unlike its effect on the original, slowly degrading AChRs, reinnervation does not modulate the degradation rate of the rapidly degrading new junctional AChRs. It merely regulates the ratio of rapidly to slowly degrading AChRs being synthesized and inserted at the NMJ.

Innervation of vertebrate muscle causes major changes in the localization and behavior of acetylcholine receptors (AChRs; see reviews by Fambrough, 1979; Salpeter and Loring, 1985; Schuetze and Role, 1987). The present study investigates the mechanism(s) whereby innervation stabilizes AChR degradation at the neuromuscular junction (NMJ).

AChRs in preinnervated embryonic muscle have a degradation half-life $\left(t_{1 / 2}\right)$ of $\sim 1 \mathrm{~d}$, while AChRs at the adult innervated NMJ have a $t_{1 / 2}$ of $\sim 8-10 \mathrm{~d}$ (for review, see Salpeter, 1987). Denervation increases the degradation rate of junctional AChRs (Levitt et al., 1980; Loring and Salpeter, 1980; Stanley and Drachmann, 1981; Brett et al., 1982; Bevan and Steinbach,

\footnotetext{
Received Apr. 12, 1990; revised July 17, 1990; accepted Aug. 17, 1990.

We thank Maria Szabo and Dorothy Bell for technical help, Tom Podleski, Edwin Salpeter, Charles McCulloch, Rick Rotundo, Zach Hall, and Daniel Wetzel for useful discussions, and Deborah Moslehi for preparing the manuscript. This work was supported by NIH Grant NS09315 and by a grant from the Cornell University Biotechnology Program sponsored by the New York State Science and Technology Foundation (a consortium of industries), the U.S. Army Research Office, and the National Science Foundation. Data was presented in preliminary form at the Society of Neuroscience in 1989.

Correspondence should be addressed to Dr. Miriam M. Salpeter, Section of Neurobiology and Behavior, Cornell University, W113 Mudd Hall, Ithaca, NY 14853.

Copyright (C) 1990 Society for Neuroscience $0270-6474 / 90 / 123905-11 \$ 03.00 / 0$
}

1983) without changing the junctional receptor density significantly, at least up to several weeks after denervation (Frank et al., 1975; Loring and Salpeter, 1980, Bader, 1981; Brenner and Rudin, 1989). However, the time course and extent of the increase in degradation rate differs in different reports. These differences could be due, in part, to the different muscles used and to the time after denervation that the studies were carried out. Systematic studies of one muscle, the mouse sternomastoid muscle, using a combination of gamma counting and transmission electron microscope (TEM) autoradiography, have established that, after denervation, there are 2 populations of AChRs with different degradation rates interspersed on the top of the junctional folds of that NMJ (Levitt and Salpeter, 1981; Shyng and Salpeter, 1989). These 2 populations of AChRs have been defined as "original AChRs" (i.e., those synthesized and inserted into the postjunctional membrane before denervation) and "new AChRs" (i.e., those synthesized and inserted into the junction after denervation).

The original AChRs are a slowly degrading population, having a $t_{1 / 2}$ of $\sim 8 \mathrm{~d}$ in innervated muscle. About 9-10 d after denervation, their degradation rate accelerates to a $t_{1 / 2}$ of $\sim 3 \mathrm{~d}$ (Levitt and Salpeter, 1981), but reverts to the predenervation rate upon reinnervation (Salpeter et al., 1986). Thus, inncrvation can modulate the degradation rate of the original AChRs in the plasma membrane. The new AChRs, on the other hand, are more rapidly degrading and predominantly have a $t_{1 / 2}$ of $\sim 1 \mathrm{~d}$ (Shyng and Salpeter, 1989). Their degradation rate is thus similar to that previously reported for AChRs in preinnervated embryonic muscle (Fambrough, 1979; Steinbach et al., 1979; Salpeter and Loring, 1985) and for extrajunctional AChRs in denervated muscle (Berg and Hall, 1975; Chang and Huang, 1975; Burden, 1977). No information has yet been published on the response of these new junctional AChRs to reinnervation. In the present study, we investigated the responses of the new AChRs to reinnervation and asked whether modulation of the degradation rate of these rapidly degrading $A C h R s$ is a likely mechanism whereby innervation regulates $A C h R$ degradation at the NMJ.

Here, we report that reinnervation does not have a significant effect on the degradation rate of the "new" junctional AChRs during most of the expected lifetime of these AChRs. These data suggest that, after innervation of muscle cells, a major aspect of the regulation of $\mathrm{AChR}$ degradation may be the replacement of the rapidly degrading by the slowly degrading population of AChRs. The possibility that denervated muscle may also synthesize some slowly degrading AChRs $\left(t_{1 / 2} \sim 3-4 \mathrm{~d}\right)$, constituting $<10 \%$ of junctional AChRs, is discussed. 


\section{Materials and Methods}

\section{Denervation}

Sternomastoid muscles from adult female white mice (CD)-1, Charles River Breeding Laboratories, Inc., Wilmington, MA) were used in this study. The muscles were exposed and denervated under Nembutal anesthesia $(60 \mathrm{mg} / \mathrm{kg}$ body weight). Denervation was done either by crushing the nerve with forceps in order to obtain rapid and synchronous reinnervation or by ligating and cutting the nerve to prevent reinnervation. Prolonged denervation was maintained by reanesthetizing the animals and recrushing the nerve every second day for 10-14 d. The state of denervation and reinnervation was determined on days 1-12 after the last crush using 2 assays. The first assay used a functional criterion, observing muscle contraction visually in response to tetanic nerve stimulation $(100 \mathrm{~Hz})$ as previously described (Fertuck et al., 1975). The second assay was more precise and used a morphological criterion. It involved the presence of nerve terminals at the endplate in electron micrographs (see Fig. 1 for criterion used). If nerve terminals were seen within $200 \mathrm{~nm}$ of the postsynaptic membrane, as in Figure $1 b$ and $c$, a junction was considered reinnervated (in a normal innervated NMJ, the primary synaptic cleft is $\sim 50 \mathrm{~nm}$ ).

\section{Labeling of AChRs}

To label AChRs inserted for any predetermined period, existing receptors were blocked by topically applying $3 \mu \mathrm{M}$ nonradioactive $\alpha$-bungarotoxin ( $\alpha$-BGT) to the muscle for $1.5 \mathrm{hr}$ (to be called "cold saturation"). We found an improved postoperative survival rate if animals were precold-saturated for $0.5 \mathrm{hr}$ on the day before cold saturation, though the reason for this is not yet clear. Also, atropine was administered (1.8 $\mathrm{mg} / \mathrm{kg}$ ) prior to the operation to help postoperative survival. Newly inserted AChRs were then labeled with ${ }^{125}$ I- $\alpha$-BGT $(3 \mu \mathrm{M}$ by topical application for $1.5 \mathrm{hr}$, to be called "hot saturation") at different times after the cold saturation. The extent of saturation was judged using TEM autoradiography after cold saturating muscles and, immediately thereafter, hot saturating them. We found that our procedure inactivated 95$98 \%$ of specific $\alpha$-BGT binding sites at the NMJ, similar to that previously reported (Loring and Salpeter, 1980; Shyng and Salpeter, 1989).

\section{Preparation of tissue for degradation studies}

Animals were killed by intracardial perfusion with $4 \%$ paraformaldehyde in phosphate buffer $(0.067 \mathrm{M} ; \mathrm{pH}, 7.4)$ at various times up to 16 d after hot saturation. Degradation rates were assessed using gamma counting (Berg and Hall, 1975; Levitt and Salpeter, 1981; Salpeter et al., 1986) or TEM autoradiography (Salpeter and Harris, 1983; Shyng and Salpeter, 1989).

Gamma counting. For endplate-specific labeling, the sternomastoid muscle was dissected and stained for acetylcholinesterase (Karnovsky and Roots, 1964) to identify the endplate band. The muscle was cut into 3 pieces containing the endplate band and the 2 adjacent nonendplate regions. Each piece was weighed, and its radioactivity was measured by a gamma counter. The endplate-specific radioactivity was determined by subtracting the nonendplate-band radioactivity from the endplate-band radioactivity on a per weight basis.

TEM autoradiography. The endplates were not stained, and the paraformaldehyde perfused tissue was postfixed in $\mathrm{OsO}_{4}$ and embedded in I.X112. The flat substrate procedure of Salpeter and Bachmann (1964) was followed, using monolayers of Ilford L4 emulsion and D19 development ( $4 \mathrm{~min}$ at $20^{\circ} \mathrm{C}$; see Fertuck and Salpeter, 1976; Salpeter, 1981). The ${ }^{125} \mathrm{I}-\alpha$-BGT binding-site density was determincd from the TEM autoradiograms

specifically at the postjunctional membrane of the NMJs (as previously described, e.g., Fertuck and Salpeter, 1976; Matthews-Bellinger and Salpeter, 1978).

\section{Determination of degradation rates}

The degradation rate of AChRs was determined by plotting the residual radioactivity $X(t)$, after labeling with ${ }^{125} I-\alpha-B G T$, as a function of time $t$ after labeling. This decay accurately reflects AChR degradation (Devreotes and Fambrough, 1975; see review by Fambrough, 1979). Because we wanted to determine the possible existence of more than 1 population of AChRs, each with its own first-order kinetics, we modeled the observed $X(t)$ by the sum, $Y(t)$, of several pure exponential decays to get the best fit. We express the decay rate for each exponential in terms of half-life ( $t_{1 / 2}$, i.e., the time for half the radioactivity to be lost). We used the least-squares method to obtain the minimum number of exponentials that would give a good fit to the data. The goodness of fit was judged by the $F$ test (Snedecor and Cochran, 1980; Seber and Wild, 1989) using the following equation:

$$
F=\left[\Sigma_{i}\left(X_{i}-Y_{i}\right)^{2} /(\mathrm{SEM})_{i}^{2}\right] /(\text { d.f. }),
$$

where $X_{i}$ is the mean of the measured radioactivity, $Y_{i}$ is the predicted value from the fitting model, and (SEM) $)_{i}$ is the standard error of $X_{i}$, all at time $t_{i}$. The degrees of freedom (d.f.) is the number of time points minus the number of parameters used in the fitting model.

The $F$ test was also used to compare the difference between the control and experimental data. In this case, $(\mathrm{SEM})_{i}{ }^{2}$ was the sum of the (SEM) ${ }^{2}$ of the control and the experimental group at time $t_{i}$.

\section{Results}

\section{Regeneration of crushed nerves}

Two assays were used to determine the time and extent of muscle reinnervation after multiple nerve crush. The physiological assay showed that tetanic nerve stimulation caused no muscle response $2 \mathrm{~d}$ after the last crush, but by $3 \mathrm{~d}$ it had already caused slight muscle contraction. The morphological assay examined TEM micrographs of at least 50 endplates (several micrographs from each endplate) randomly chosen from more than 2 muscles for each of the critical time points (i.e., 1, 2, 3, 4, 5, 6, 9, 10, and $12 \mathrm{~d}$ after the last crush). As with the physiological criterion, no reinnervation was seen 1 and $2 \mathrm{~d}$ after the last nerve crush, and reinnervated endplates were first seen in the experimental muscles $3 \mathrm{~d}$ after the last crush (of a total of 50 endplates, 35\% were reinnervated by day 3 ). The onset of reinnervation is therefore between day 2 and 3 after multiple crushing. By day 6, all of 50 randomly chosen endplates showed reinnervation $(\sim 10 \%$ of the images were as seen in Fig. $1 b$, i.e., nerve terminal within $200 \mathrm{~nm}$, and $\sim 90 \%$ were as seen in Fig. $1 c$, i.e., nerve terminal within $50 \mathrm{~nm})$. Endplates from later time points $(9,10$, and 12 d) were all again fully reinnervated. Poisson statistics gives $P(r)$ $=\left(\mu^{r} / r !\right) e^{-\mu}$ for the probability of observing $r$ denervated (or innervated) endplates if the expected number is $\mu$. Using this equation, we calculate that the probability that even $10 \%$ of the cndplatcs are still not being reinncrvated on day 6 is $<0.01$. By contrast, in control animals whose nerves were cut and ligated, more than 100 endplates were examined between days 1 and 16 after denervation, and none had terminal nerve fibers, showing only images as in Figure $1 a$. The probability that even $5 \%$ of the control endplates were innervated is less than 0.01 . Both the physiological and morphological reinnervation time course reported here is in good agreement with that previously reported by Rich and Lichtman (1989), but slightly faster than that previously reported after only a single nerve crush (Salpeter et al., 1986).

Effect of reinnervation on degradation of "new" junctional AChRs inserted into NMJ of denervated muscle: Group I

Three experimental paradigms were used, as shown in Figure $2, a-c$. The experimental paradigm for Group I is given in Figure $2 a$. In this group of experiments, all preexisting AChRs wcre cold saturated at the time of the first nerve crush, and the nerves were recrushed every second day for $10 \mathrm{~d}$. The new AChRs were hot saturated $12 \mathrm{~d}$ after cold saturation (i.e., $2 \mathrm{~d}$ after the last crush, and thus before any reinnervation was seen in the experimental animals). In the control animals, nerves were ligated and cut at time of the last crush to prevent regeneration. 

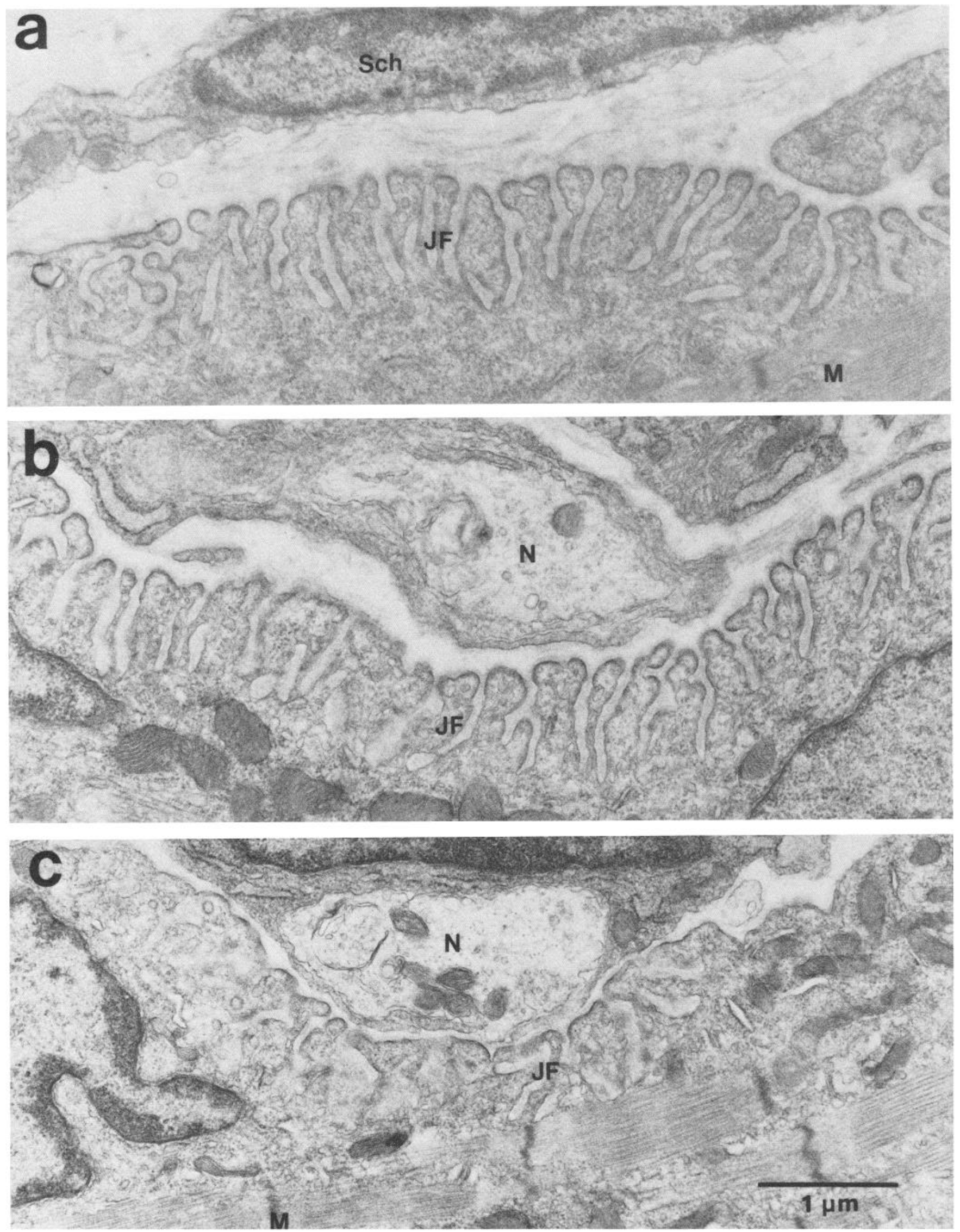

Figure 1. Fine structure of denervated and reinnervated NMJs. $a$, A denervated endplate showing no evidence of reinnervation. $b$, An endplate being reinnervated showing nerve terminal in vicinity (within $200 \mathrm{~nm}$ ) of the postsynaptic membrane. $c$, A fully reinnervated endplate. Both $b$ and $c$ profiles were judged as reinnervated. By day 6 after the last nerve crush, $\sim 10 \%$ of the endplate profiles fall in the category of $b$, and $\sim 90 \%$, in the category of $c . J F$, junctional folds; $M$, muscle; $N$, nerve terminal; $S c h$, Schwann cell. Magnification, 26,000×. 
The degradation curves of AChRs in reinnervated and control muscles of Group I, obtained by gamma counting, are given in Figure $3 a$. Using the $F$ test, we found no siznificant difference between the experimental and control curves $(p>0.25)$. The slight but not significant deviation from control beyond $10 \mathrm{~d}$ after full reinnervation will be discussed below. From this analysis, it appears that reinnervation does not have an effect on the degradation of new AChRs.

\section{Presence of Slow Component}

A surprising result in the data from Group $I$ is that neither the experimental nor the control curve can be fit well by a single exponential. When fit to a single exponential, $t_{1 / 2}$ values of $\sim 2$ $\mathrm{d}$ were obtained for both the control and experimental curves, but with a poor fit $(F$ test, $p<0.05$ ). For the experimental data, this could mean either that more than 1 population with different degradation rates are contributing to the data, or that there may be a shift from one degradation rate to another in a single population of AChRs. In the control data, only the first alternative is reasonable. Using the least-squares analysis (as described in Materials and Methods), an excellent fit to the control data was obtained with 2 components $(F$ test, $p>0.25$ ). Figure $3 b$ shows these 2 components: a major one with a $t_{1 / 2}$ of $1.1 \mathrm{~d}\left(74 \%\right.$ of total) and a minor one with a $t_{1 / 2}$ of $3.6 \mathrm{~d}(26 \%$ of total). Interestingly, the 2 half-lives obtained are those previously sccn in $\mathrm{AChR}$ populations at endplates of denervated muscle. The $t_{1 / 2}$ of $\sim 1 \mathrm{~d}$ was that of the new AChRs inserted after denervation (Shyng and Salpeter, 1989), and the $t_{1 / 2}$ of $\sim 3-$ $4 \mathrm{~d}$ was that of the accelerated original AChRs (Levitt and Salpeter, 1981; Salpeter et al., 1986). No improvement in the fit was obtained when adding a third component $\left(t_{1 / 2}\right.$ of $\left.8 \mathrm{~d}\right)$. Thus, in addition to the rapidly degrading new AChRs $\left(t_{1 / 2}\right.$ of $\sim 1$ d) previously described (Shyng and Salpeter, 1989), the hotlabeled pool of control data contains a small amount of a slow component $\left(t_{1 / 2} \sim 3-4 \mathrm{~d}\right)$.

\section{Nature of the slow component}

Because the nerve in the control animal was not allowed to regenerate, the 3-d slow component can not be due to reinnervation. As will be seen below, several sources can contribute to this slow component. Three of these are artifacts due to residual original AChRs contaminating the hot-saturated pool: (1) The first is due to incomplete initial cold saturation of the original AChRs. However, as indicated in Materials and Methods, less than $5 \%$ of AChRs were not cold saturated and therefore would have decayed to $\ll 1 \%$ of the hot-saturated pool by the time of hot saturation. (2) The second artifact is due to unbinding of the nonradioactive $\alpha$-BGT from the cold-saturated original AChRs during the $12 \mathrm{~d}$ between cold and hot saturation ( $t_{1 / 2}$ for unbinding, $\sim 36 \mathrm{~d}$; Bevan and Steinbach, 1983). The unbound original AChRs would have accelerated to a $t_{1 / 2}$ of $3-4$ $\mathrm{d}$ by $10 \mathrm{~d}$ after the initial crush and would then be hot saturated together with the new AChRs. Using Equation 2 given in the Appendix, we calculate that the contribution to the slow component due to unbinding in Group I constitutes 9\% of the hotlabeled pool. (Note that the problem introduced by unbinding of nonradioactive $\alpha$-BGT between cold and hot saturation in this study is different from that introduced by unbinding of radioactive $\alpha$-BGT during the time that degradation is measured, which only slightly underestimates the true $t_{1 / 2}$ of degradation; e.g., see Bevan and Steinbach, 1983; Salpeter et al., 1986.) (3) A third source of contamination could be a delay between the initial nerve crush (when cold saturation was performed) and the time when rapidly degrading new $\mathrm{AChRs}$ first appear. Any original AChRs inserted during this delay would be part of the hot-saturated pool. The equations in the Appendix indicate that, if such a delay exists, there would be an additional contribution of $9 \%$ for a $3-d$ delay or $6 \%$ for a $2-d$ delay. Three days is the maximum it could be without it having been detected by Shyng and Salpeter (1989), and $2 \mathrm{~d}$ would be compatible with the first appearance of increased AChR mRNA after denervation (Goldman et al., 1988).

The calculated value for the percent of the labeled pool expected to be slow $\left(t_{1 / 2}\right.$ of $\left.3 \mathrm{~d}\right)$ due to contamination by residual original AChRs was thus $9 \%$ if no delay exists and, at most, $18 \%$ with a $3-d$ delay. This leaves at least $8 \%$ unaccounted for.

We suggest that the additional source could be a de novo synthesis of some slowly degrading AChR $\left(t_{1 / 2} \sim 3-4 d\right)$ in denervated muscle. Note that, throughout this study, we refer to AChRs degrading with a $t_{1 / 2}$ of $\sim 3-4 \mathrm{~d}$ as slowly degrading or the slow component. This is by comparison with AChRs with a $t_{1 / 2}$ of $1 \mathrm{~d}$ and because, as will be seen in the Discussion, we believe that the AChRs with $t_{1 / n}$ of 3-4 d in the denervated muscle are like the accelerated original AChRs.

Figure $3 c$ gives an alternative display of the fast component for the experimental group. This is achieved by subtracting the slow component (as described in Fig. $3 c$, caption). The remaining population of the AChRs degrades with a single exponential, having a $t_{1 / 2}$ of $\sim 1 \mathrm{~d}$, for at least $12 \mathrm{~d}$ after the last crush $(6 \mathrm{~d}$ after full reinnervation). Figure $3 c$ thus graphically confirms the conclusion drawn from Figure $3 a$ that reinnervation does not alter the degradation rate of the rapidly degrading new AChRs for more than 6 half-lives.

\section{Groups II and III: decreasing slow component confirms} results from Group I

From the data in Group I (Fig. 3a-c), we concluded that innervation does not affect the degradation rate of rapidly degrading new AChRs. In addition, we saw a small slow component in our labeled pool. Experimental Groups II and III (paradigms shown in Fig. $2 b, c$ ) were designed to predictably decrease the extent of the slow component and thus to test the conclusion rcached from Group I. Group II was designed primarily to decrease any part of the slow component due to the delay in insertion of new AChRs. To achieve this, cold saturation was performed $4 \mathrm{~d}$ after the first nerve crush, a time longer than any expected time delay before rapidly degrading new AChRs should begin to accumulate. All AChRs inserted during that time delay will thus be cold saturated and will not contaminate the hot pool. As in Group I, the nerve was recrushed every second day, but for a total of $14 \mathrm{~d}$ rather than 10 $\mathrm{d}$ after the first crush (leaving the time between cold and hot saturation in Groups I and II the same, but the total time after initial nerve crush $4 \mathrm{~d}$ longer in Group II). The AChRs were again hot saturated $2 \mathrm{~d}$ after the last crush (i.e., $16 \mathrm{~d}$ after the first crush). Prolonging the time between the initial denervation and hot saturation was also expected to decrease the artifactual slow components, as it allowed more time for the original AChRs to degrade. This would happen rapidly beyond $10 \mathrm{~d}$ after initial crush because any original AChRs would be degrading with a $t_{1 / 2}$ of $3 \mathrm{~d}$ after that time (Levitt and Salpeter, 1981). Using Equation 1, given in the Appendix, we calculated that, in Group II, the slow component due to contamination by residual orig- 
(a) Group I

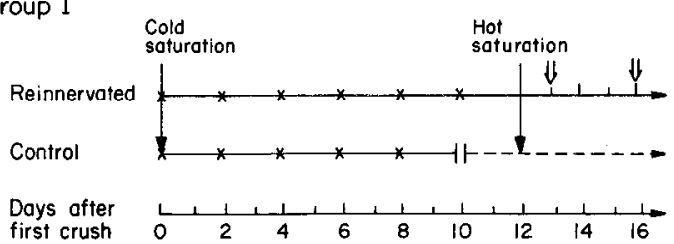

(b) Group II

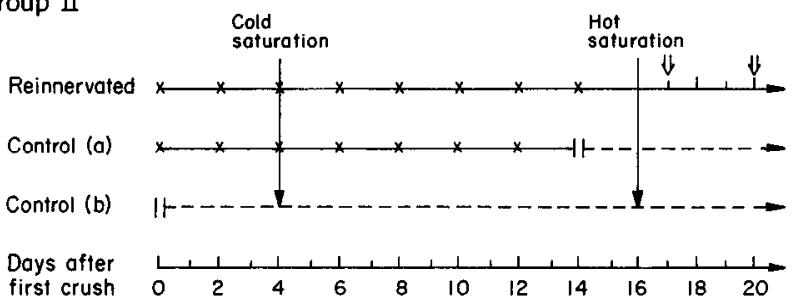

(c) Group III
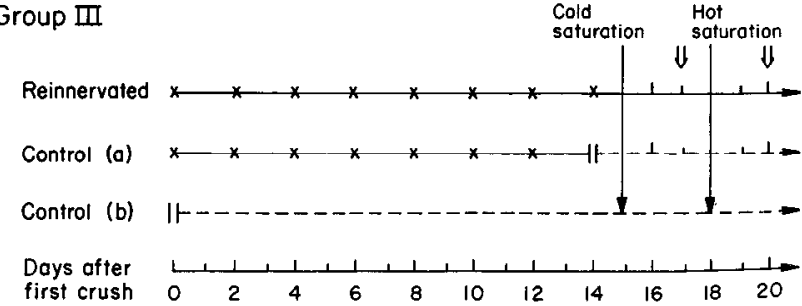

Figure 2. Experimental paradigm of Groups I, II, and III. Times at which the nerve crush was performed are marked by $\times$; nerve cut and ligation marked by $\|$. Open arrows mark the time during which the reinnervation process occurs. Time of cold saturation and hot saturation are labeled. Nerves were allowed to regenerate after the last nerve crush in the experimental (reinnervated) muscles, but not in the control muscles. In one control group (Control a), the nerves were crushed every second day for up to $10 \mathrm{~d}$ after the cold saturation, as in the experimental muscles, but at the time of the last crush, the control nerves were ligated and cut to prevent nerve regeneration. An additional control group, in which the nerves were ligated and cut without being crushed, was done in Groups II and III (Control b). (Cold and hot saturation of control muscles were performed at the same time as that of experimental muscles.) $a$, Group I. Cold saturation was performed at the time of the first nerve crush, and hot saturation was performed $2 \mathrm{~d}$ after the last crush (i.e., $12 \mathrm{~d}$ after cold saturation and the first nerve crush). $b$, Group II. Cold saturation was performed $4 \mathrm{~d}$ after the first nerve crush, and hot saturation was performed $2 \mathrm{~d}$ after the last crush (i.e., $12 \mathrm{~d}$ after cold saturation, $16 \mathrm{~d}$ after the first nerve crush). $c$, Group III. Cold saturation was performed $1 \mathrm{~d}$ after the last nerve crush (i.e., $15 \mathrm{~d}$ after the first crush), and hot saturation was performed $3 \mathrm{~d}$ thereafter.

Figure 3. a, Degradation curves obtained by gamma counting in reinnervated (solid circles) and control (open circles) muscles of Group I. Three to 8 animals were used for each time point. Error bars represent SEM. Time after hot saturation is given on the lower horizontal axis, and time after last nerve crush is on the top horizontal axis. Reinnervation, marked by the open arrows, occurred between days 1 and 4 after hot saturation (i.e., beginning by day 3 and completed by day 6 after the last nerve crush). No significant difference between the experimental and control curve can be observed $(P<0.25)$. However, the experimental curve deviates slightly from the control after day $8 . b$, Analysis of control curve (open circles) using least-squares fit shows that the curve can be best fit by a $74 \%$ fast component with a $t_{1 / 2}$ of $1.1 \mathrm{~d}$ (dashed-anddotted line) and a $26 \%$ slow component with a $t_{1 / 2}$ of $3.6 \mathrm{~d}$ (broken line). The sum of the 2 exponentials (solid curve) shows a good fit to the observed control data ( $F$ test, $P>0.25$ ). $c$, If the slow component of the control curve represents residual original AChRs that had accelerated to a $t_{1 / 2}$ of $3 \mathrm{~d}$ in the denervated muscle (see text), it would be expected to decelerate in response to reinnervation with the time course previously seen by Salpeter et al. (1986). When this deceleration of the slow component is calculated, the dotted curve is obtained, shown here overlying the experimental data of the reinnervated group (solid circles).
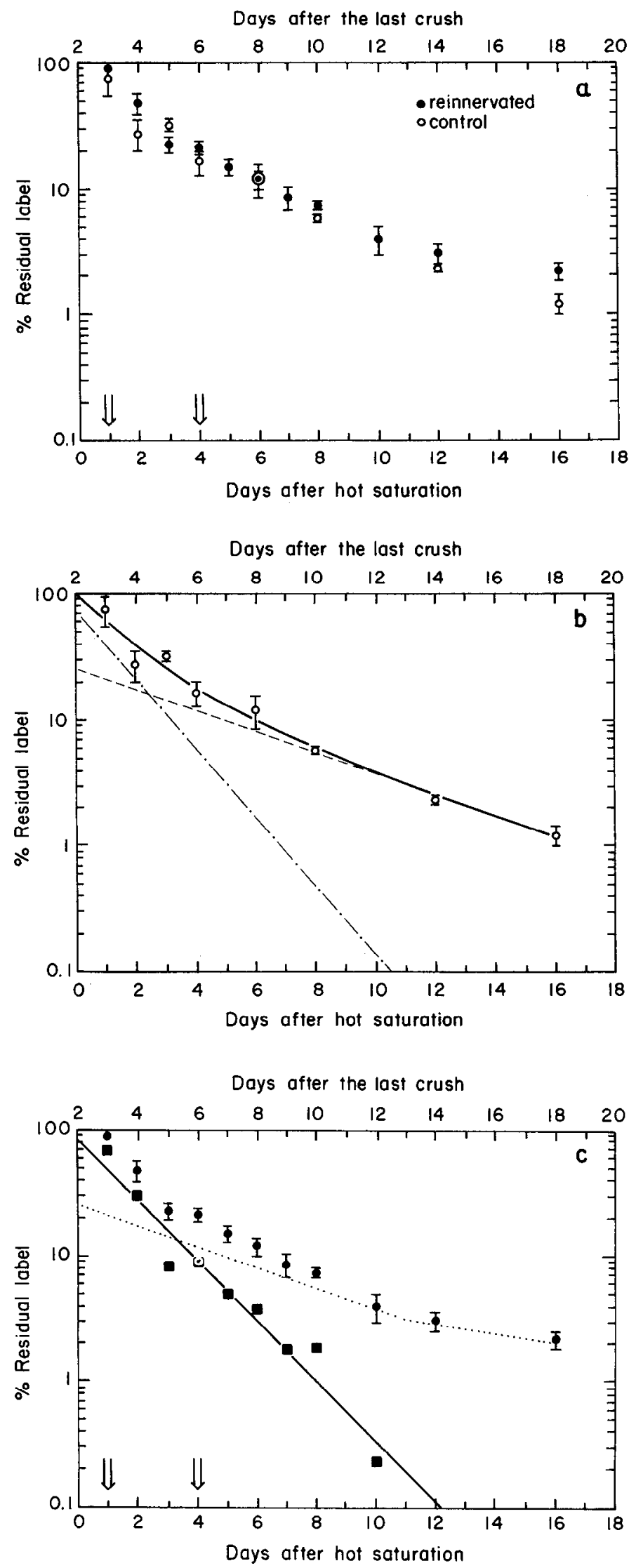

When the amount of this slow component is subtracted from the experimental reinnervated data, the remaining data consists of a single fast component (squares) with a $t_{1 / 2}$ of $1.2 \mathrm{~d}$, which is unaltered by reinnervation. 

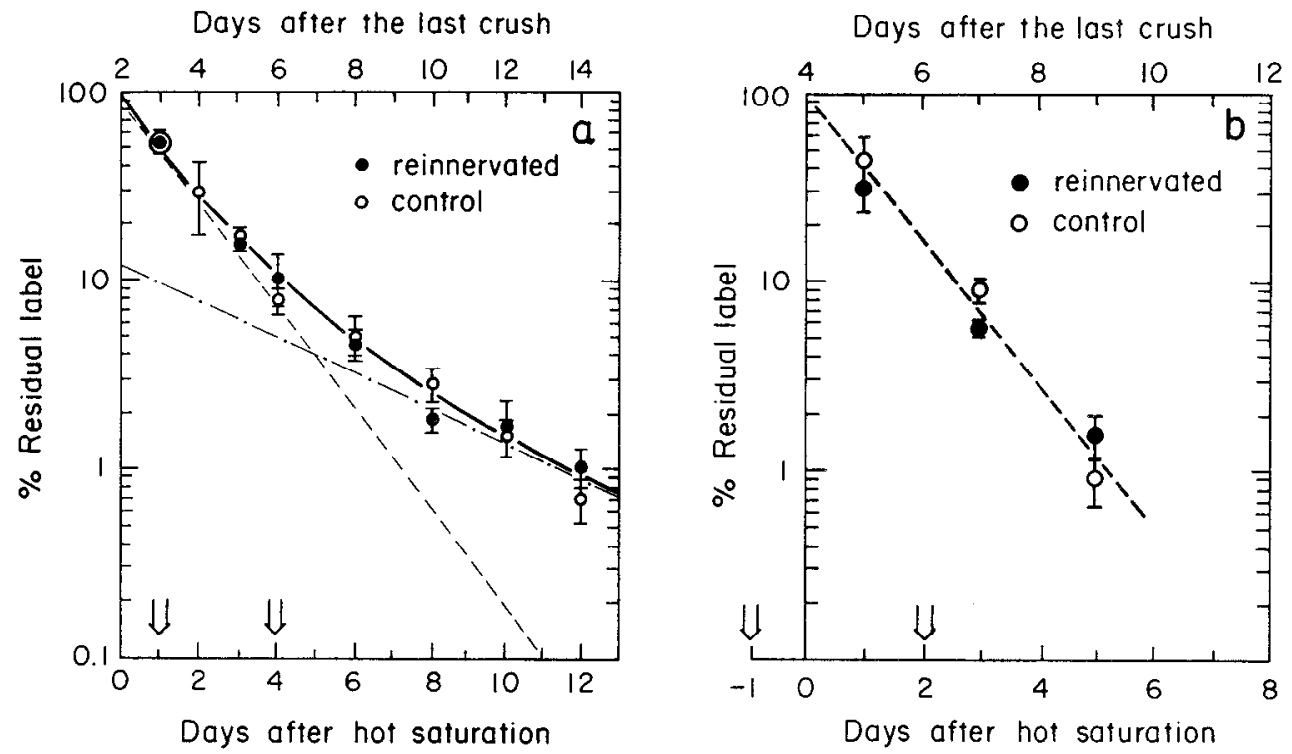

Figure 4. Degradation curves obtained for AChRs in Group II (a) and Group III $(b)$ and determined by gamma counting in reinnervated (solid circles) and control (open circles) muscles. The 2 control groups (see Fig. 2) were combined because they were not significantly different. Three to 5 animals were used for each time point. Error bars represent SEM. The beginning and end of the reinnervation process are marked by open arrows. There is no significant difference between the experimental and the control curve $(p>0.25)$ for either group. $a$, For Group II, fitting of the control curve by the method of least squares showed the presence of a fast component (dashed line) consisting of $89 \%$ with a $t_{1 / 2}$ of $0.9 \mathrm{~d}$ and a slow component (dashed-and-dotted lines) consisting of $11 \%$ of total hot pool with a $t_{1 / 2}$ of $3.3 \mathrm{~d}$. The sum of the fast and slow components (solid line) shows a good fit to the data $\left(F\right.$ test, $p>0.25$ ). $b$, For Group III, the dashed line shows the best fit of the data points $\left(t_{1 / 2} \sim 1 \mathrm{~d}\right)$ for the combined reinnervated and control data between days 1 and 5 after hot saturation (5-9 d after the last crush). No change is seen in response to reinnervation (reinnervation time marked by open arrows) for at least 3 half-lives after full regeneration or 6 half-lives after initiation of regeneration, by which time the labcl is down to $<2 \%$ of the initial value and is close to the background. An expected slow component $\left(t_{1 / 2} \sim 3 \mathrm{~d}\right)$ of $\sim 4 \%$ would be degraded to $\sim 1 \%$ by $5 \mathrm{~d}$ after hot saturation and would not be easily detectable.

inal AChRs due to unbinding or delay should be only 4 or $3 \%$ of the hot-saturated pool with or without a 3-d time delay, respectively. Thus, the Group II protocol greatly decreases the contribution to the slow component due to any residual original AChRs. This protocol should not however affect any slow component due to de novo synthesis in the denervated muscle.

Group III (see Fig. 2c) was designed to decrease most of the slow component, independent of its source. This would eliminate the need to perform curve fitting and subtraction of the control slow component and would allow us to determine directly whether reinnervation affected the degradation rate of the rapidly degrading new AChRs. The period between cold and hot saturation was decreased to $3 \mathrm{~d}$. Nerves were multiply crushed for $14 \mathrm{~d}$ but AChRs were not cold saturated until $1 \mathrm{~d}$ after the last crush and were hot saturated $3 \mathrm{~d}$ later. (We therefore labeled AChRs that were accumulated between days 15 and 18 after the initial denervation.) This procedure could decrease the slow component for several reasons. Any slow component due to unbinding of cold toxin is decreased because (1) most of the AChRs being cold saturated at this time are already of the 1-d rapidly degrading species, and (2) not much unbinding will occur in $3 \mathrm{~d}$. (One can calculate, using the equations given in the Appendix, that the artifactual slow component due to residual original AChRs using this cxpcrimental paradigm is expected to be only about $1 \%$ of the hot-saturated pool.) Moreover, if there is a de novo source of AChRs with a $t_{1 / 2}$ of $\sim 3-4 \mathrm{~d}$, having only a 3-d insertion period will decrease the amount of those AChRs being inserted to half its steady-state number.

Figure 4, $a$ and $b$, show the degradation curves for Groups II and III, respectively. Using the same analysis as for Group I, we found that, in both Groups II and III, the expcrimental and control data were not significantly different. Thus, by using a protocol that eliminates the need for the subtraction method, Group III directly demonstrates the unresponsiveness of the rapidly degrading new AChRs to reinnervation, for a period longer than the lifetime of most (99-88\%) of these receptors. We also performed a least-squares analysis to obtain the minimum number of components to fit the data. In Group II, the best fit was obtained with an $89 \%$ fast component $\left(t_{1 / 2}=0.9 \mathrm{~d}\right)$ and an $11 \%$ slow component $\left(t_{1 / 2}=3.3 \mathrm{~d} ; F\right.$ test, $\left.p>0.25\right)$. Because the artifactual source of the slow component was expected to contribute only $3-4 \%$, we again have $7-8 \%$ of the hot pool unaccounted for. As with Group I, when the 3-d slow component from the control group was subtracted from the experimental curve, a rapidly degrading population of AChRs with a $t_{1 / 2}$ of $\sim 1.3 \mathrm{~d}$ was seen for up to $\sim 14 \mathrm{~d}$ after the last crush unaffected by reinnervation (data not shown). In Group III, a single component ( $t_{1 / 2}$ of $\left.0.8 \mathrm{~d}\right)$ gave the best fit $(p>0.25)$, though the expected slow component of about $3-5 \%$ could not be excluded because that would have been degraded to less than $1 \%$ by the end of the time course in Figure $4 b$.

\section{TEM autoradiography}

We used TEM autoradiography to gain assurance that our results obtained by gamma counting were valid for junctional AChRs and not influenced by extrajunctional contaminants. Because we define junctional AChRs as those located on the junctional folds of an NMJ, the endplate-specific label (see Materials and Methods) obtained by gamma counting may contain some extrajunctional label that develops in a gradient around the NMJ 
carly after denervation (Levitt-Gilmour and Salpeter, 1986). It was previously shown by TEM autoradiography that, in denervated muscle, the new junctional AChRs have a degradation half-life of $\sim 1 \mathrm{~d}$ (Shyng and Salpeter, 1989), similar to that of the postdenervation extrajunctional AChRs (e.g., Chang and Huang, 1975). Thus, if innervation does not slow the degradation of the new junctional AChRs, the gamma-counting results should give an accurate value for the half-life of these AChRs. However, if innervation should slow the degradation of the new junctional AChRs while the extrajunctional AChRs remain rapidly degrading, the gamma-counting results may dilute the effect on junctional AChRs. Therefore, as an additional test, TEM autoradiography was performed on muscles from Group I taken 3, 4, 9, 10, and $12 \mathrm{~d}$ after the last crush. The results given in Table 1 show that the same $t_{1 / 2}$ and proportion of slowly degrading AChRs is present on the postjunctional folds of the NMJ as is seen in the total endplate-specific band by gamma counting. Thus, the presence of extrajunctional AChRs did not distort the results on the degradation rate of the junctional AChRs. This strengthens the conclusion that reinnervation did not selectively affect the degradation rate of the AChRs at the junction. Furthermore, if we divided the TEM autoradiograms of days 3 and 4 after the last crush (and thus the period during which reinnervation was occurring) into 2 groups, one with and one without regenerated nerves, as seen in Figure 5, the residual $\mathrm{AChR}$ site densities and thus the degradation rates were the same in the 2 groups. The ratios of $\alpha$-BGT site density in the reinnervated endplates to that in the denervated endplates were $1.04 \pm 0.03$ and $1.06 \pm 0.01$ for days 3 and 4 after the last crush, respectively. (A similar subdivision could not be performed for the TEM autoradiograms at the later times, i.e., 9,10 , and $12 \mathrm{~d}$ after the last crush, because at those times the nerves were fully regenerated.)

\section{Discussion}

During development or after denervation of adult muscle, innervation results in the accumulation of slowly degrading AChRs $\left(t_{1 / 2} \sim 8-10 \mathrm{~d}\right)$ at the NMJ (Steinbach et al., 1979; Reiness et al., 1981; Steinbach, 1981; Weinberg et al., 1981). In this study, we emphasize that, to understand the possible mechanisms involved, one has to consider separately the responses of 2 populations of AChRs. These 2 populations of AChRs, original and new, are predominantly synthesized in innervated or denervated muscles, respectively. They are interspersed on the postjunctional membrane of denervated NMJs (Shyng and Salpeter, 1989), yet maintain different degradation rates.

Salpeter et al. (1986) have previously shown that reinnervation decelerates the degradation of any original AChRs still remaining at the NMJ. In the present study, we report that a similar change upon reinnervation was not observed in the degradation rate of new AChRs. Therefore, reinnervation has, in effect, a different physiological effect on the degradation rates of the 2 populations of junctional AChRs. Whereas deceleration of the degradation rate occurred within less than 2 half-lives after full reinnervation of the original AChRs, no deccleration of the degradation rate of the rapidly degrading new AChRs was seen for more than 3 half-lives after full reinnervation (by which time, very few of the AChRs remain). Because reinnervation is not fully synchronous and more than $30 \%$ of the AChRs are already innervated between day 2 and 3 after the last crush, a considerable number of AChRs were, in effect, followed for

$\begin{aligned} & \text { Table 1. Comparison between the TEM autoradiographic and the } \\
& \text { gamma-counting results on the degradation rate of junctional AChRs } \\
& \text { of Group I }\end{aligned}$
\begin{tabular}{lll} 
Days \\
after \\
the last & TEM autoradiographic data & Gamma-counting data \\
nerve & (\% residual site density & (\% residual label \\
crush & normalized to day 3 ) & normalized to day 3 ) \\
\hline 3 & $100.0 \pm 10.0$ & $100.0 \pm 2.0$ \\
4 & $58.9 \pm 4.8$ & $55.4 \pm 0.4$ \\
9 & $12.2 \pm 5.3$ & $9.1 \pm 3.1$ \\
10 & $12.4 \pm 0.6$ & $10.0 \pm 0.1$ \\
12 & $6.8 \pm 0.3$ & $5.3 \pm 1.1$ \\
\hline
\end{tabular}

The error is the total range of data from 2 animals.

more than 6 half-lives after reinnervation, and yet, no deviation from the $t_{1 / 2}$ of $1 \mathrm{~d}$ was seen.

From our data, we cannot conclude that the rapidly degrading AChRs are incapable of having their degradation rate altered by reinnervation, if given enough time. We do conclude, however, that a switch in degradation rate does not occur within a time scale that could make it a plausible mechanism whereby the nerve stabilizes the degradation rate of junctional AChRs.

\section{Reclassification of junctional AChRs}

Based on our current results, we propose to classify the junctional AChRs not only by when they appear in the membrane (as was done previously in the definitions of the "original" AChRs as being present prior to denervation and "new" AChRs inserted after denervation; Levitt and Salpetcr, 1981; Shyng and Salpeter, 1989), but also by what their degradation rate is and by how they respond to innervation. We define (1) a slowly degrading population of AChRs (to be called $\mathrm{R}_{s}$ ), whose degradation rate accelerates in the plasma membrane (from a $t_{1 / 2}$ of $\sim 8 \mathrm{~d}$ to one of $\sim 3 \mathrm{~d}$ ) in response to denervation and decelerates again (from a $t_{1 / 2}$ of $\sim 3 \mathrm{~d}$ to one of $\sim 8 \mathrm{~d}$ ) after reinnervation and (2) a rapidly degrading population of AChRs (to be called $\mathrm{R}_{r}$ ), whose degradation rate retains a $t_{1 / 2}$ of $\sim 1$ d for a minimum of 3 halflives after reinnervation.

One has to bear in mind that the absolute values of the degradation rates of the $\mathrm{AChR}$ populations and the time course of their modulation might not be identical in all muscles. For example, AChRs in embryonic muscle, cloned muscle cell lines, and the extrajunctional region of denervated muscle have a degradation half-life ranging from 11 to $35 \mathrm{hr}$ (Fambrough, 1979; Neugebauer et al., 1985; Salpeter and Loring, 1985). Also, original AChRs in diaphragm muscle accelerate by $\sim 4 \mathrm{~d}$ after denervation (Stanley and Drachman, 1981; Brett et al., 1982; Bevan and Steinbach, 1983; Wetzel and Salpeter, 1990), whereas those of the sternomastoid muscle do not accelerate until $\sim 9$ $10 \mathrm{~d}$ after denervation (Levitt and Salpeter, 1981; Salpeter et al., 1986). After denervation of the mouse soleus muscles, the original AChRs accelerate to a slightly faster rate $\left(t_{1 / 2} \sim 2 \mathrm{~d}, \mathrm{M}\right.$. M. Salpeter, unpublished observations). Thus, the degradation rate of the accelerated original $\mathbf{R}_{s}$ may approach that of the $\mathbf{R}_{r}$. Yet, the postulate of $2 \mathrm{AChR}$ populations $\mathbf{R}_{s}$ and $\mathbf{R}_{r}$, whose degradation rates may be differently regulated, makes it conceptually easier to explore possible mechanisms for that regulation. 

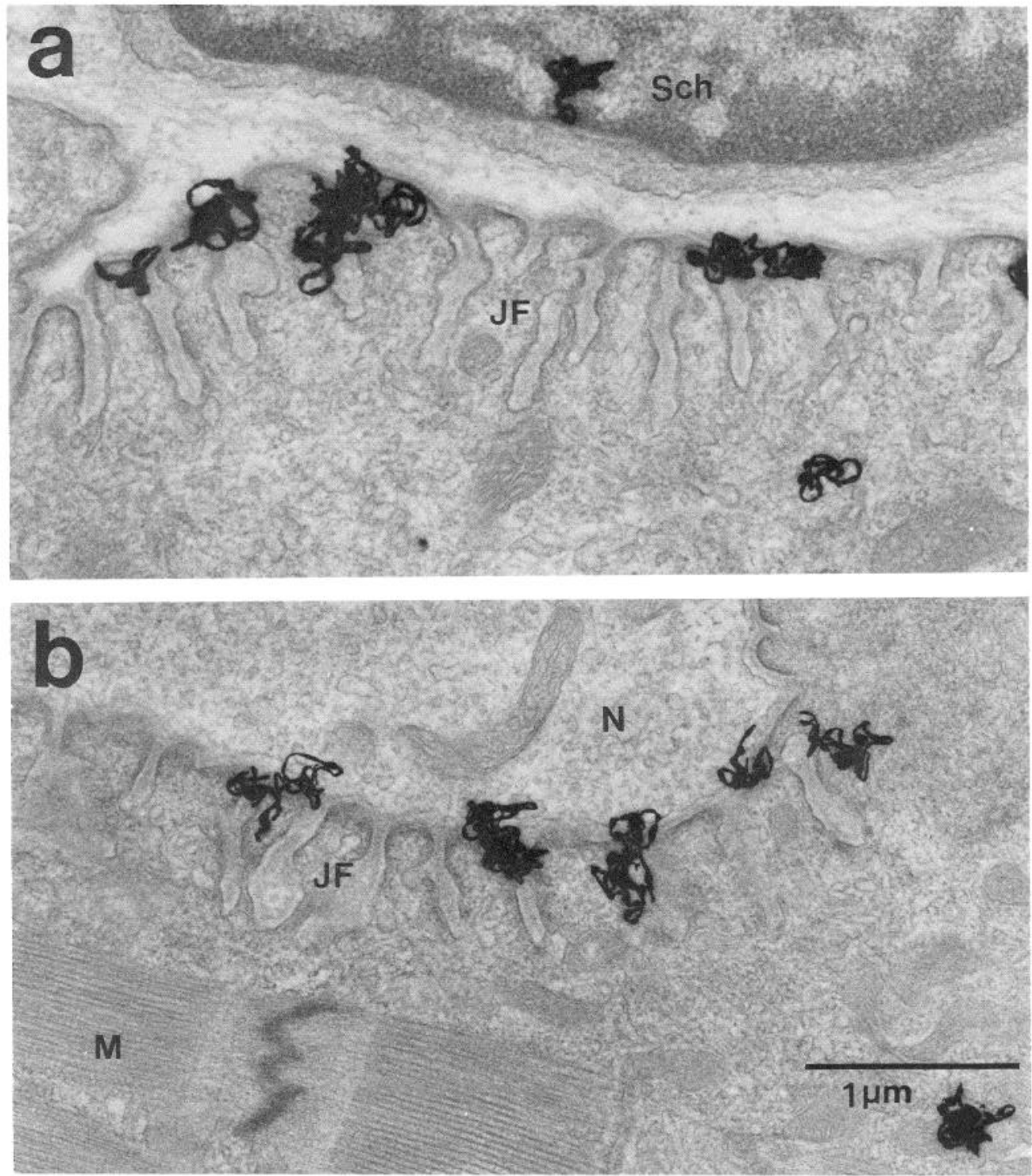

Figure 5. TEM autoradiograms of prereinnervated endplate $(a)$ and reinnervated endplate $(b)$. On days 3 and 4 after the last nerve crush, when reinnervation had begun but was not yet completed, the site-density ratio of reinnervated to still-denervated endplate profiles was analyzed. The site-density ratios are $1.04 \pm 0.03$ and $1.06 \pm 0.01$ for day 3 and 4 , respectively. $J F$, junctional folds; $M$, muscle; $N$, nerve terminal; $S c h$, Schwann cell. Magnification: $28,000 \times$.

\section{Molecular structure and degradation rate of AChRs}

Because the control of the AChR degradation by the nerve begins during the synthesis of the AChR, the molecular structure of the AChR is likely to be relevant. Interestingly, there is great similarity between neural regulation of the degradation of junctional AChRs, as seen in the present study, and that of the channel properties of the AChRs as described by Brehm and Kullberg (1987) and Henderson et al. (1987). It is therefore tempting to suggest that the ratio of $\mathrm{R}_{r}$ to $\mathbf{R}_{s}$ is somehow related to the ratio of the $\gamma$ and $\epsilon \mathrm{AChR}$ subunits, as is the regulation of AChR channel properties (Mishina et al., 1986; Witzemann et al., 1987; Brehm and Henderson, 1988; Brenner and Rudin, 1989). The early literature had indicated that the time course of appearance of the high-conductance AChR at NMJs differs somewhat from that of the appearance of the slowly degrading AChRs (see reviews by Salpeter and Loring, 1985; Salpeter,
1987; Schuetze and Role, 1987). This would suggest that the 2 AChR properties (gating and degradation) cannot both be regulated simply by the difference in the $\gamma$ and $\epsilon$ subunits. However, the earlier studies may need to be modified, or other transcriptional differences and posttranslational modifications may be involved. One possibility would be a posttranslational modification that allows the degradation rates of the $\mathbf{R}_{s}$ population to be further modulated (accelerated and decelerated) after insertion in the plasma membrane. Because the rodent $\epsilon$ subunit has now been cloned (Buonanno et al., 1989; Camacho et al., 1989), direct studies on the degradation rate of $\epsilon$-containing AChRs in transformed cells should resolve the role of that subunit in AChR degradation. If $\epsilon$ and $\gamma$ are involved in determining the $\mathbf{R}_{s}: \mathbf{R}_{r}$ ratio, then we predict that the $\epsilon$-containing AChRs in noninnervated cells would degrade only about $2-4$ times slower than the $\gamma$-containing cells, that is, the difference between the $t_{1 / 2}$ of the $\mathbf{R}_{r}$ and the accelerated $\mathbf{R}_{s}$ seen in denervated muscles. How- 
ever, only the $\epsilon$-containing AChRs should respond to innervation by decelerating to a $t_{1 / 2}$ of $\sim 8 \mathrm{~d}$. After this paper was submitted, the first part of this prediction was confirmed by $\mathrm{Gu}$ et al. (1990). They transfected COS cells to express AChR containing either the $\epsilon$ or the $\gamma$ subunit and found that the $\epsilon$-containing AChRs degrade about a factor of 2 slower than the $\gamma$-containing AChRs. It remains to be determined whether only the degradation rate of the $\epsilon$-containing AChRs can bc modulated by innervation.

\section{$R_{\mathrm{r}}$ and $R_{\mathrm{s}}$ in denervated and innervated muscle}

The $\mathbf{R}_{s}$ and $\mathbf{R}_{r}$ populations are predominantly present in innervated and denervated muscles, respectively. We suggest that the state of innervation determines the ratio of these 2 populations being synthesized, yet both populations may be synthesized in either innervated or noninnervated muscle. Our calculations (using equations given in the Appendix) and the overlap in the results from Groups I and II lead us to conclude that, in denervated muscle, a very small percentage $(\sim 8 \%)$ of newly inserted junctional AChRs may be $\mathrm{R}_{s}$ with a $t_{1 / 2}$ of $\sim 3-4 \mathrm{~d}$. If the $\mathrm{R}_{s}$ is the $\epsilon$-containing AChR, our results are consistent with reports that some $\epsilon$-containing AChRs are present in denervated muscle long after all original AChRs should have degraded (Brenner and Sakmann, 1983; Henderson et al., 1987; Gu and Hall, 1988a; Brenner et al., 1990). It is of interest that high conductance (and thus $\epsilon$-containing) AChRs have been found to constitute $\sim 7 \%$ of total AChR in denervated muscle (Witzemann et al., 1987) and thus equal to our $\mathbf{R}_{s}$ population.

In innervated junctions, a small, rapidly degrading population $(\sim 10 \%)$ of AChRs at the NMJ has been described by Stanley and Drachman (1983, 1987; see also Ramsay et al., 1988). We and others (e.g., Bevan and Steinbach, 1983) also occasionally saw a fast component during the first day after labeling innervated AChRs when gamma counting was used. TEM autoradiographic studies tend not to show this fast component at the NMJ (e.g., Salpeter and Harris, 1983), and its nature is not yet clear.

We also conclude that there probably is a 2-3-d delay before $\mathbf{R}_{r}$ are first inserted. A similar delay is seen after denervation in the initiation of increased AChR mRNA and in the appearance of the $\gamma$ subunit (Goldman et al., 1988) and may in fact account for the delay proposed by us.

\section{Mechanisms for switch in degradation rates after reinnervation}

Two distinct mechanisms could cause junctional AChRs to develop a slow degradation rate after innervation. The first mechanism could involve slowing the degradation rate of $\mathrm{AChRs}$ already in the membrane. So far, this mechanism has been demonstrated only for the accelerated original AChRs (i.e., for $\mathbf{R}_{s}$ receptors; Salpeter et al., 1986) and could therefore operate only if reinnervation occurs rapidly before all the original $\mathrm{AChRs}$ have been degraded. Contrary to this suggestion, Drachman and colleagues (Stanley and Drachman, 1983, 1987; Ramsay et al., 1988) suggest that a rapidly degrading population of AChRs might be slowed by the nerve and serve as precursors for the larger population of stable AChRs. In the present study, the $\mathbf{R}_{r}$ population does not decelerate even after 3-6 half-lives following reinnervation, by which time these AChRs are already degraded to $\ll 10 \%$. If only $10 \%$ of $\mathrm{R}_{r}$ switched to $\mathrm{R}_{s}$, the steadystate AChR population in the membrane would contain about $50 \% \mathrm{R}_{r}$. To our knowledge, no one has reported such a high ratio of $\mathrm{R}_{r}: \mathrm{R}_{s}$ at innervated NMJs. The fast component usually constitutes only about $10 \%$ of the population. Our data, plus this argument, suggest that slowing the degradation rate of $\mathbf{R}_{r}$ is not the most likely physiological mechanism whereby the nerve stabilizes the degradation rate of junctional AChRs, unless fully mature neurons behave differently than newly regenerated ones.

The second mechanism could involve replacing the rapidly degrading $\left(R_{r}\right)$ AChRs $\left(t_{1 / 2}\right.$ of $\left.1 \mathrm{~d}\right)$ by newly synthesized slowly degrading $\left(\mathbf{R}_{s}\right)$ AChRs (cf. Brenner and Rudin, 1989). This second mechanism could result from a preferential downregulation of the synthesis of the $\mathbf{R}_{r}$ population, analogous to the differential regulation in synthesis of the "embryonic"-type $(\gamma)$ subunit and the "adult"-type ( $\epsilon$ ) subunit after innervation (Henderson et al., 1987; Witzemann et al., 1987; Brehm and Henderson, 1988; Gu and Hall, 1988a,b; Brenner et al., 1990).

Both of the above-mentioned mechanisms for regulating degradation of junctional AChRs may be mediated, in part, by muscle activity. For the first mechanism, which involves modulating the degradation rate of original AChRs, Avila et al. (1989) have shown that original AChRs can be accelerated when muscles are inactivated by botulinum toxin or $\alpha$-BGT, and Brenner and Rudin (1989) reported that electrical activity prevents the acceleration of original AChRs after denervation. To definitively establish that electrical activity mimics innervation of original AChRs, it would also be necessary to show that electrical activity can stabilize original AChRs back to a $t_{1 / 2}$ of $8 \mathrm{~d}$ after they have accelerated to a $t_{1 / 2}$ of $\sim 3 \mathrm{~d}$.

For the second mechanism, which involves replacing the $\mathbf{R}_{r}$ by $\mathbf{R}_{s}$, it is well known that muscle activity can downregulate AChR synthesis (Fambrough, 1979; Klarsfeld and Changeux, 1985; Fontaine et al., 1987; Goldman et al., 1988), possibly providing for a selective downregulation of the rapidly degrading AChRs relative to the slowly degrading ones. A recent study by Fumagalli et al. (1990) can be interpreted in that light. After prolonged denervation, when most original AChRs had been replaced by new AChRs, the muscle was stimulated for $15 \mathrm{~d}$. When the stimulation period was completed, the AChRs were labeled, and the degradation rate was found to be as in innervated muscles $\left(t_{1 / 2}>8 \mathrm{~d}\right)$. These results could mean either that the degradation rate of the new $\Lambda$ ChRs had been stabilized as they suggest, or that, during the $15-\mathrm{d}$ stimulation period, all the rapidly degrading AChRs had been replaced by slowly degrading AChRs (as our model suggests). To determine definitively whether the rapidly degrading AChRs could indeed be stabilized by electrical activity, these $R_{r}$ would have to be labeled prior to the initiation of electrical stimulation.

In summary, our studies suggest that the degradation rate of junctional AChRs could involve neural control during the synthesis of both $\mathbf{R}_{r}$ and $\mathbf{R}_{s}$ as well as after insertion of $\mathbf{R}_{s}$ into the postsynaptic membrane. The neural role in producing an NMJ with slowly degrading AChRs probably involves both the stabilization of $\mathbf{R}_{s}$ in the membrane and the replacement of $\mathrm{R}_{r}$ by $\mathrm{R}_{s}$. Although there is evidence to support this hypothesis, a direct proof clearly requires further research at the molecular level, where correlations between degradation behavior and receptor structure can be studied.

\section{Appendix}

One can calculate the fraction, $f$, of hot-saturated slow AChRs to total hot-saturated rapid plus slow AChRs, due to the unbinding of the nonradioactive $\alpha$-BGT, the lack of full initial cold 
saturation, or a delay in the onset of the rapidly degrading AChR insertion, as follows:

We define the following terms:

$\begin{aligned} t_{0}= & \text { the time of the first nerve crush. } \\ t_{c}= & \text { the time of cold saturation. } \\ t_{h}= & \text { the time of hot saturation. } \\ = & \text { the time at which the rapidly degrading AChRs start } \\ & \text { being inserted into the NMJ. } \\ T_{1}= & \text { the degradation half-life }\left(t_{1 / 2}\right) \text { of the slow AChRs. }\end{aligned}$

$T_{1}=$ the that the slow AChRs can have 2 different values for $T_{1}$. These are $8 \mathrm{~d}$ for $10 \mathrm{~d}$ after the first nerve crush and $3 \mathrm{~d}$ thereafter (Levitt and Salpeter, 1981).

$T_{2}=$ the $t_{1 / 2}$ of the rapidly degrading AChRs, which is $1 \mathrm{~d}$.

$A=$ all junctional $\mathrm{AChRs}$ present at time of the first nerve crush $\left(t_{0}\right)$. The total number of $A C h R s$ are assumed to stay constant throughout and can be normalized to 1 .

$A^{\prime}=$ fraction of $A$ that is slow at time $t_{c}$ and has not yet degraded by time $t_{h}=\left[2^{-\left(t_{c}-t_{0}\right) / T_{1}}\right]\left[2^{-\left(t_{h}-t_{c}\right) / T_{1}}\right]$

$A^{\prime \prime}=$ the fraction of $A$ that has been replaced by rapidly degrading AChRs by time $t_{c}$ but has not yet degraded by time $t_{h}=\left[1-2^{-\left(t_{c}-t_{\mathrm{dcla} y}\right) / T_{1}}\right]\left[2^{-\left(t_{h}-t_{c}\right) / T_{2}}\right]$.

$B=$ the fraction of cold-saturated AChRs that have become unbound by time $t_{h}$ (the $t_{1 / 2}$ for unbinding is $36 \mathrm{~d}$; Bevan and Steinbach, 1983) $=1-2^{-\left(t_{h}-t_{c} / 36\right.}$.

$C=$ fraction of AChRs that are blocked by nonradioactive $\alpha$-BGT at time $t_{c}$ (experimentally, it was determined to be $>95 \%$ ).

$D=[(1-C)+B C]$, that is, the fraction of AChRs that were present at time $t_{c}$ that are available for hot saturation due either to lack of full cold saturation or to unbinding during the period between cold and hot saturation.

In the case when $t_{\text {delay }} \leq t_{c}$,

$$
f=\frac{A^{\prime} D}{1-\left(A^{\prime}+A^{\prime \prime}\right)+\left(A^{\prime} D+A^{\prime \prime} D\right)} .
$$

When $t_{\text {delay }} \geq t_{c}$, the formula is somewhat more complex, requiring an added term, $A_{\text {add }}$. Then

$$
f=\frac{A_{\text {add }}^{\prime}+A^{\prime} D}{R+A_{\text {add }}^{\prime}+A^{\prime} D}
$$

where $A^{\prime}{ }_{\text {add }}=\left[1-2^{-\left(t_{\text {delay }}-t_{c}\right) / T_{1}}\right]\left[2^{-\left(t_{h}-t_{\text {detay }}\right) / T_{1}}\right]$, and $R$ is the amount of rapid AChRs available at time $t_{h}=1-2^{-\left(t_{h}-t_{\text {delay }}\right) / T_{1}}$. (Note: from $t_{\text {delay }}$ to day $10, T_{1}=8 \mathrm{~d}$, and from day 10 to $t_{h}, T_{1}=3$ d.)

\section{References}

Avila OL, Drachman DB, Pestronk A (1989) Neurotransmission regulates stability of acetylcholine receptors at the neuromuscular junction. J Neurosci 9:2902-2906.

Bader D (1981) Density and distribution of $\alpha$-bungarotoxin binding sites in postsynaptic structures of regenerated rat skeletal muscle. J Cell Biol 88:338-345.

Berg DK, Hall ZW (1975) Loss of $\alpha$-bungarotoxin from junctional and extrajunctional acetylcholine receptors in rat diaphragm in vivo and in organ culture. J Physiol (Lond) 252:771-789.

Bevan S, Steinbach JH (1983) Denervation increases the degradation rate of acetylcholine receptors at end-plates in vivo and in vitro. $\mathrm{J}$ Physiol (Lond) 336:159-177.

Brchm P, Henderson L (1988) Ricgulation of acctylcholinc receptor channel function during development of skeletal muscle. Dev Biol 129:1-11.

Brehm P, Kullberg R (1987) Acetylcholine receptor channels on adult mouse skeletal muscle are functionally identical in synaptic and nonsynaptic membrane. Proc Natl Acad Sci USA 84:2550-2554.

Brenner HR, Rudin W (1989) On the effect of muscle activity on the end-plate membrane in denervated mouse muscle. J Physiol (Lond) 410:501-512.

Brenner HR, Sakmann B (1983) Neurotrophic control of channel properties at neuromuscular synapses of rat muscle. J Physiol (Lond) 337 : 159-171.

Brenner HR, Witzemann V, Sakmann B (1990) Imprinting of acetylcholine receptor messenger RNA accumulation in mammalian neuromuscular synapses. Nature 344:544-547.

Brett RS, Younkin SG, Konieczkowski M, Slugg RM (1982) Accelerated degradation of junctional acetylcholine receptor- $\alpha$-bungarotoxin complexes in denervated rat diaphragm. Brain Res 233:133142.

Buonanno A, Mudd J, Merlie JP (1989) Isolation and characterization of the $\beta$ and $\epsilon$ subunit genes of mouse muscle acetylcholine receptor. J Biol Chem 264:7611-7616.

Burden SJ (1977) Acetylcholine receptors at the neuromuscular junction: developmental changes in receptor turnover. Dev Biol 61:7985.

Camacho P, Goodman RH, Mandel G, Brehm P (1989) Isolation of a cDNA encoding the epsilon subunit of the rat muscle AChR. Biophys J 55:554a.

Chang CC, Huang MC (1975) Turnover of junctional and extrajunctional acetylcholine receptors in the rat diaphragm. Nature 253:643644

Devreotes PN, Fambrough DM (1975) Acetylcholine receptor turnover in membranes of developing muscle fiber. J Cell Biol 65:335358.

Fambrough D (1979) Control of acetylcholine receptors in skeletal muscle. Physiol Rev 59:165-227.

Fertuck HC, Salpeter MM (1976) Quantitation of junctional and extrajunctional acetylcholine receptors by electron microscope autoradiography after I-125- $\alpha$-bungarotoxin binding at mouse neuromuscular junctions. J Cell Biol 69:144-158.

Fertuck HC, Woodward WW, Salpeter MM (1975) In vivo recovery of muscle contraction after $\alpha$-bungarotoxin binding. J Cell Biol 66 : 209-213.

Fontaine B, Klarsfeld A, Changeux JP (1987) Calcitonin gene-related peptide and muscle activity regulate acetylcholine receptor $\alpha$-subunit mRNA levels by distinct intracellular pathways. J Cell Biol 105:13371342.

Frank E, Gautvik K, Sommerschild H (1975) Persistence of junctional AChR following dencrvation. Cold Spring Harbor Symp Quant Biol 40:275-281.

Fumagalli G, Balbi S, Cangiano A, Lomo T (1990) Regulation of turnover and number of acetylcholine receptors at neuromuscular junctions. Neuron 4:563-569.

Goldman D, Brenner HR, Heinemann S (1988) Acetylcholine receptor $\alpha-, \beta, \gamma-$, and $\delta$-subunit mRNA levels are regulated by muscle activity. Neuron 1:329-333.

Gu Y, Hall Z (1988a) Immunological evidence for a change in subunits of the acetylcholine receptor in developing and denervated rat muscle. Neuron 1:117-125.

Gu Y, Hall ZW (1988b) Characterization of acetylcholine receptor subunits in developing and in denervated mammalian muscle. J Biol Chem 263:12878-12885.

Gu Y, Franco A Jr, Gardner PD, Lansman JB, Forsayeth JR, Hall Z (1990) Properties of embryonic and adult muscle acetylcholine receptors transiently expressed in cos cells. Neuron 5:147-157.

Henderson LP, Lechleiter J, Brehm P (1987) Single channel properties of newly synthesized acetylcholine receptors following denervation of mammalian skeletal muscle. J Gen Physiol 89:999-1014.

Karnovsky MJ, Roots L (1964) A "direct-coloring" thiocholine method for cholinesterase. J Histochem Cytochem 12:219-221.

Klarsfeld A, Changeux JP (1985) Activity regulates the levels of acetylcholine receptor $\alpha$-subunit mRNA in cultured chicken myotubes. Proc Natl Acad Sci USA 82:4558-4562.

Levitt TA, Salpeter MM (1981) Denervated endplates have a dual population of junctional acetylcholine receptors. Nature 291:239241

Lcvitt TA, Loring RH, Salpeter MM (1980) Neuronal control of acetylcholine receptor turnover rate at a vertebrate neuromuscular junction. Science 210:550-551.

Levitt-Gilmour TA, Salpeter MM (1986) Gradient of extrajunctional 
acetylcholine receptors early after denervation of mammalian muscle. J Neurosci 6:1606-1612.

Loring R, Salpeter MM (1980) Denervation increases turnover rate of junctional acetylcholine receptors. Proc Natl Acad Sci USA 77: 2293-2298.

Matthews-Bellinger J, Salpeter MM (1978) Distribution of acetylcholine receptors at frog neuromuscular junctions with a discussion of some physiological implications. J Physiol (Lond) 279:197-213.

Mishina M, Takai T, Imoto K, Noda M, Takahashi T, Numa S, Methfessel C, Sakmann B (1986) Molecular distinction between fetal and adult forms of muscle acetylcholine receptor. Nature 321:406-441.

Neugebauer K, Salpeter MM, Podleski TR (1985) Differential responses of L5 and rat primary muscle cells to factors in rat brain extract. Brain Res 346:58-69.

Ramsay DA, Drachman DB, Pestronk A (1988) Rapid synthesis of acetylcholine receptors at neuromuscular junctions. Brain Res 462: 134-141.

Reiness CG, Weinberg CB, Hall ZW (1981) Metabolic slabilization of acetylcholine receptors at newly formed neuromuscular junctions in rat. Dev Biol 84:247-254.

Rich MM, Lichtman JW (1989) In vivo visualization of pre- and postsynaptic changes during synapse elimination in reinnervated mouse muscle. J Neurosci 9:1781-1805.

Salpeter MM (1981) High resolution autoradiography. In: Techniques in the life sciences, "Techniques in cellular physiology," Pt I, Vol P1/I (P106), pp. 1-45. Flsevier North Holland.

Salpeter MM (1987) Development and neural control of the neuromuscular junction and of the junctional acetylcholine receptor. In: The vertebrate neuromuscular junction (Salpeter MM, ed), pp 55115. New York: Liss.

Salpeter MM, Bachmann L (1964) Autoradiography with the electron microscope, a procedure for improving resolution, sensitivity and contrast. J Cell Biol 22:469-477.

Salpeter MM, Harris R (1983) Distribution and turnover rate of acetylcholine receptors throughout the junction folds at a vertebrate neuromuscular junction. J Cell Biol 96:1781-1785.

Salpeter MM, Loring RH (1985) Nicotinic acetylcholine receptors in vertebrate muscle: properties, distribution and neural control. Prog Neurobiol 25:297-325.
Salpeter MM, Cooper DL, Levitt-Gilmour T (1986) Degradation rates of acetylcholine receptors can be modified after they are inserrted into the postjunctional plasma membrane of the vertebrate neuromuscular junction. J Cell Biol 103:1399-1403.

Schuetze E, Role L (1987) Developmental regulation of nicotinic acetylcholine receptors. Annu Rev Neurosci 10:403-457.

Seber GAF, Wild CJ (1989) Nonlinear regression. New York: Wiley.

Shyng SL, Salpeter MM (1989) Degradation rate of acetylcholine receptors inserted into denervated vertebrate neuromuscular junctions. J Cell Biol 108:647-651.

Snedecor GW, Cochran WG (1980) Statistical methods, 7th ed. Iowa State University Press.

Stanley EF, Drachman DB (1981) Denervation accelerates the degradation of junctional acetylcholine receptors. Exp Neurol 73:390396.

Stanley EF, Drachman DB (1983) Rapid degradation of "new" acetylcholine receptors at neuromuscular junctions. Science 222:67-69.

Stanley EF, Drachman DB (1987) Stabilization of acetylcholine receptors at neuromuscular junctions: analysis by specific antibodies. Ann NY Acad Sci 505:121-132.

Steinbach JH (1981) Developmental changes in acetylcholine receptor aggregates at rat skeletal neuromuscular junctions. Dev Biol 84:267276.

Steinbach JH, Merlie J, Heinemann S, Bloch R (1979) Degradation of junctional and extrajunctional acetylcholine receptors by developing rat skeletal muscle. Proc Natl Acad Sci USA 76:3547-3551.

Weinberg CB, Sanes JR, Hall ZW (1981) Formation of neuromuscular junctions in adult rats: accumulation of acetylcholine receptors, acetylcholinesterase and components of synaptic basal lamina. Dev Biol 84:255-266.

Wetzel DM, Salpeter MM (1990 Fibrillation and accelerated AChR degradation in long term muscle organ culture. Muscle \& Nerve, in press.

Witzemann V, Barg B, Nishikawa Y, Sakmann B, Numa S (1987) Differential regulation of muscle acetylcholine receptor $\gamma$ - and $\epsilon$-subunit mRNAs. FEBS Lett 223:104-112. 